\title{
Clinical outcomes after whole-genome sequencing in patients with metastatic non-small-cell lung cancer
}

\author{
Erica S. Tsang, ${ }^{1}$ Yaoqing Shen, ${ }^{2}$ Negar Chooback, ${ }^{1}$ Cheryl Ho, ${ }^{1}$ Martin Jones, ${ }^{2}$ \\ Daniel J. Renouf, ${ }^{1}$ Howard Lim, ${ }^{1}$ Sophie Sun, ${ }^{1}$ Stephen Yip, ${ }^{3}$ Erin Pleasance, ${ }^{2}$ \\ Diana N. Ionescu, ${ }^{3}$ Karen Mungall, ${ }^{2}$ Katayoon Kasaian, ${ }^{2}$ Yussanne $\mathrm{Ma}_{1}{ }^{2}$ \\ Yongjun Zhao, ${ }^{2}$ Andrew Mungall, ${ }^{2}$ Richard Moore, ${ }^{2}$ Steven J.M. Jones, ${ }^{2}$ \\ Marco Marra, ${ }^{2,4}$ and Janessa Laskin ${ }^{2}$ \\ ${ }^{1}$ Division of Medical Oncology, ${ }^{2}$ Canada's Michael Smith Genome Sciences Center, BC Cancer, Vancouver, \\ British Columbia V5Z 4E6, Canada; ${ }^{3}$ Department of Pathology and Laboratory Medicine, ${ }^{4}$ Department of \\ Medical Genetics, University of British Columbia, Vancouver, British Columbia V6T 1Z4, Canada
}

Corresponding author: jlaskin@bccancer.bc.ca

(C) 2019 Tsang et al. This article is distributed under the terms of the Creative Commons Attribution-NonCommercial License, which permits reuse and redistribution, except for commercial purposes, provided that the original author and source are credited.

Ontology terms: lung

adenocarcinoma

Published by Cold Spring Harbor Laboratory Press

doi:10.1101/mcs.a002659

\begin{abstract}
The Personalized Onco-Genomics (POG) program at BC Cancer integrates wholegenome (DNA) and RNA sequencing into practice for metastatic malignancies. We examined the subgroup of patients with metastatic non-small-cell lung cancer (NSCLC) and report the prevalence of actionable targets, treatments, and outcomes. We identified patients who were enrolled in the POG program between 2012 and 2016 who had a tumor biopsy and blood samples with comprehensive DNA (80x,40× normal) and RNA sequencing followed by in-depth bioinformatics to identify potential cancer drivers and actionable targets. In NSCLC cases, we compared the progression-free survival (PFS) of "POG-informed therapies" with the PFS of the last regimen prior to POG (PFS ratio). In 29 NSCLC cases, 11 were male (38\%), the median age was $60.2 \mathrm{yr}$ (range: 39.4-72.6), and histologies included were adenocarcinoma (93\%) and squamous cell carcinoma (7\%). Potential molecular targets (i.e., cancer drivers including TP53 mutations) were identified in 26 (90\%), and $21(72 \%)$ had actionable targets. Therapies based on standard-of-care mutation analysis, such as EGFR mutations, were not considered POG-informed therapies. Thirteen received POG-informed therapies, of which three had no therapy before POG; therefore a comparator PFS could not be obtained. Of 10 patients with POG-informed therapy, median PFS ratio was 0.94 (IQR 0.2-3.4). Three (30\%) had a PFS ratio $\geq 1.3$, and three (30\%) had a PFS ratio $\geq 0.8$ and $<1.3$. In this small cohort of NSCLC, $30 \%$ demonstrated longer PFS with POG-informed therapies. Larger studies will help clarify the role of whole-genome analysis in clinical practice.
\end{abstract}

[Supplemental material is available for this article.]

\section{INTRODUCTION}

Lung cancer remains a prominent cause of cancer-related mortality, with an estimated 243,000 new cases and 162,500 deaths in the United States in 2016 alone (Siegel et al. 2016). Within Canada, there were more than 20,000 deaths attributed to lung cancer in 2016 (Canadian Cancer Society 2016). The majority of cases are classified as non-smallcell lung cancer (NSCLC), and advances in genomic analysis have altered the treatment landscape for patients with specific somatic mutations (Johnson et al. 2014). 
Advancements in genomic technology and the recognition of the role of mutations and genetic aberrations in cancer biology have led to the development of trials examining the role of molecular profiling in treatment decisions and outcomes (Stratton et al. 2009; MacConaill and Garraway 2010; Simon and Roychowdhury 2013). Cancer centers around the world have created programs to incorporate and apply these genomic technologies in the oncology clinic (Andre et al. 2012; Tsimberidou et al. 2012; Lindsay et al. 2015). Although the majority of cancer centers, including our provincial cancer care institute the BC Cancer, utilize hotspot gene panels to help guide routine clinical care, the Personalized Onco-Genomics (POG) program is a novel and unique research initiative that integrates both whole-genome (DNA) and RNA sequencing into oncology practice for metastatic malignancies providing information above and beyond the common cancer panels (Laskin et al. 2015). Using an interdisciplinary approach, potential actionable targets are identified and help to guide decision-making for the treating clinician. In this study, we examined the subgroup of patients with metastatic NSCLC, focusing on the prevalence of actionable targets, treatments, and outcomes.

\section{RESULTS}

During the study period from 2012 to 2016, 40 patients with metastatic NSCLC were enrolled in the POG program. Overall, 11 cases were excluded, of which five deteriorated clinically before POG results became available, and thus actionability could not be assigned for these individuals (see Fig. 1 for CONSORT diagram). It should be noted that in some cases, the data itself could be considered actionable, but for that particular patient it may not have been acted upon if they had clinically deteriorated or had a contraindication to a specific therapy. The added complexity of this extra layer of clinical applicability needs to be taken into account when data sharing or when considering the population impact of any intervention.

The median age at diagnosis was $60.2 \mathrm{yr}$ (IQR: 50.5-64.6), and 11 (38\%) were male. ECOG performance status at time of POG biopsy was $0(n=8), 1(n=20)$, and $2(n=1)$.

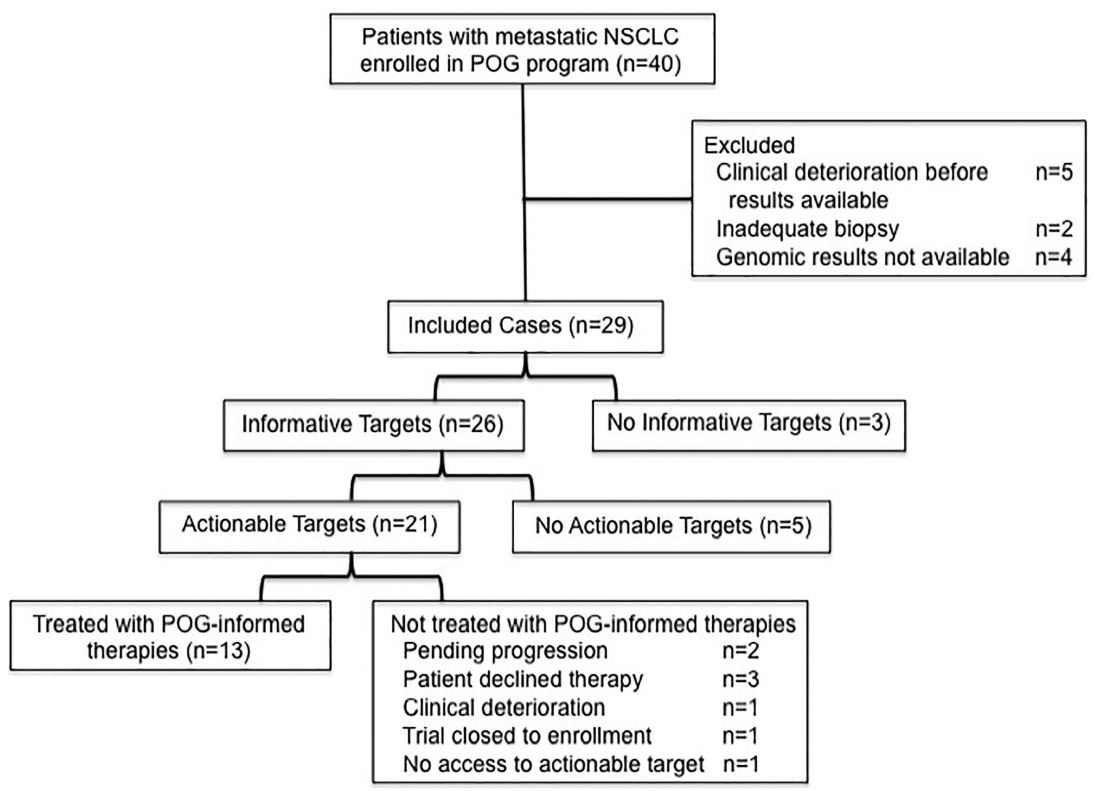

Figure 1. CONSORT diagram illustrating all POG patients with metastatic non-small-cell lung cancer (NSCLC). 
Histologies included were adenocarcinoma $(n=27)$ and squamous cell carcinoma $(n=2)$. Seventy-six percent of patients were initiated on first-line systemic therapy prior to their POG biopsy, which included chemotherapy $(n=22)$ and targeted agents $(n=2)$. Median PFS on first-line systemic therapy measured 8.20 mo (IQR: 4.9-13.5). Baseline clinicopathologic characteristics are detailed in Table 1.

Of 29 patients who underwent comprehensive analysis, 26 (90\%) had informative findings. In five cases, none of the informative findings were actionable. For instance, one patient had a ROS1 rearrangement, which was deemed informative but not actionable at that particular time. As new treatments are developed over time this finding would become actionable for another patient in the future. Of 21 patients with potentially actionable targets, 13 received POG-guided therapies, whereas eight did not: pending progression $(n=2)$, declined therapy $(n=3)$, clinical deterioration $(n=1)$, trial closed to enrollment $(n=1)$, and no access to actionable target $(n=1$; Fig. 1). Three patients who received POG-guided therapies received no therapy prior to POG; therefore, a PFS ratio could not be calculated for this cohort (Table 2). Of 10 patients with POG-informed therapy, median PFS ratio was 0.94 (IQR: $0.2-3.4$ ). Three (30\%) had a PFS ratio $\geq 1.3$, and three (30\%) had a PFS ratio $\geq 0.8$ and $<1.3$ (Fig. 2). No significant correlation was demonstrated between PFS ratio and number of lines of prior therapy or time from diagnosis to POG-guided therapy. Previous therapies are detailed in Table 3. Median OS among patients who received POG-guided therapies measured $43.53 \mathrm{mo}$ (95\% Cl: 18.28-68.8).

Three patients went on to receive second-line POG-informed therapy (Table 4). Using the previous systemic therapy prior to POG as a baseline for the PFS ratio, two (67\%) patients had a PFS ratio $\geq 1.3$ and overall median PFS ratio was 1.43 . No patients received third-line therapy.

Based on genomic analysis and actionable targets, two patients were enrolled in clinical trials, six received standard therapy, and five received therapy with an off-label indication. It

Table 1. Baseline clinicopathologic characteristics of 29 patients with metastatic non-small-cell lung cancer (NSCLC) enrolled in POG program

\begin{tabular}{lc}
\hline Baseline characteristics & $N(\%)$ \\
\hline Age (years) & 60.2 (IQR: 50.5-64.6) \\
Median (IQR) & $18(62)$ \\
Gender & $11(38)$ \\
Female & \\
Male & $8(28)$ \\
ECOG performance status & $20(69)$ \\
0 & $1(3)$ \\
1 & $27(93)$ \\
2 & $2(7)$ \\
Histology & \\
Adenocarcinoma & $22(76)$ \\
Squamous cell carcinoma & $2(7)$ \\
First-line systemic therapy & $5(17)$ \\
Chemotherapy & \\
Targeted agent & $8.20(4.9-13.5)$ \\
No first-line therapy & \\
First-line systemic therapy (months) & \\
Median PFS (IQR) & \\
\hline
\end{tabular}




\begin{tabular}{|c|c|c|c|c|c|c|c|c|}
\hline Patient & $\begin{array}{l}\text { Lines of } \\
\text { therapy } \\
\text { before } \\
\text { POG }\end{array}$ & Informative aberrations & $\begin{array}{l}\text { Actionable } \\
\text { aberrations }\end{array}$ & $\begin{array}{l}\text { Tier of } \\
\text { evidence }\end{array}$ & $\begin{array}{l}\text { POG- } \\
\text { informed } \\
\text { therapy }\end{array}$ & $\begin{array}{l}\text { Time to } \\
\text { POG- } \\
\text { informed } \\
\text { therapy } \\
\text { (months) }\end{array}$ & $\begin{array}{l}\text { PFS for } \\
\text { POG- } \\
\text { informed } \\
\text { therapy } \\
\text { (months) }\end{array}$ & $\begin{array}{l}\text { PFS } \\
\text { ratio }\end{array}$ \\
\hline 1 & 3 & $\begin{array}{l}\text { AKT2 (copy gain), EGFR } \\
\text { (p.T790M, L858R), } \\
\text { PIK3R2 (copy loss), } \\
\text { MET (overexpression) }\end{array}$ & $\begin{array}{l}\text { AKT2 (copy gain), } \\
\text { EGFR (p.T790M, } \\
\text { L858R), PIK3R2 } \\
\text { (copy loss), MET } \\
\text { (overexpression) }\end{array}$ & 3 & $\begin{array}{l}\text { Clovis } \\
\text { compound } \\
1686\end{array}$ & 70.73 & 3.77 & 0.19 \\
\hline 2 & 1 & $\begin{array}{l}\text { VEGFA (copy gain, } \\
\text { overexpression) }\end{array}$ & $\begin{array}{l}\text { VEGFA (copy gain, } \\
\text { overexpression) }\end{array}$ & 1 & Bevacizumab & 2.90 & 9.67 & 1.11 \\
\hline 3 & 1 & $\begin{array}{l}\text { NOTCH1 } \\
\text { (overexpression), } \\
\text { SDC4-NRG1 (fusion) }\end{array}$ & $\begin{array}{l}\text { NOTCH1 } \\
\text { (overexpression), } \\
\text { SDC4-NRG1 } \\
\text { (fusion) }\end{array}$ & 3 & $\begin{array}{l}\text { Phase } 1 \\
\text { NOTCH } \\
\text { trial }\end{array}$ & 14.93 & 7.40 & 0.84 \\
\hline 4 & 2 & $\begin{array}{l}\text { PI3KCA (copy gain), MET } \\
\text { (copy gain, } \\
\text { overexpression) }\end{array}$ & $\begin{array}{l}\text { MET (copy gain, } \\
\text { overexpression), } \\
\text { P13KCA (copy gain), } \\
\text { mutational burden }\end{array}$ & 1 & Nivolumab & 65.40 & 1.07 & 0.16 \\
\hline 5 & 3 & $\begin{array}{l}\text { EGFR (copy gain), ERBB2 } \\
\text { (overexpression), } \\
\text { ERBB3 } \\
\text { (overexpression), PTEN } \\
\text { (underexpression) }\end{array}$ & $\begin{array}{l}\text { EGFR (copy gain), } \\
\text { ERBB2 } \\
\text { (overexpression), } \\
\text { ERBB3 } \\
\text { (overexpression) }\end{array}$ & 1 & $\begin{array}{l}\text { Everolimus } \\
\text { and } \\
\text { erlotinib }\end{array}$ & 25.90 & 1.87 & 0.10 \\
\hline 6 & 1 & EML4-ALK (fusion) & EML4-ALK (fusion) & 1 & Crizotinib & 2.17 & 30.70 & $16.75^{\mathrm{a}}$ \\
\hline 7 & 3 & $\begin{array}{l}\text { EML4-ALK (fusion), ROS1 } \\
\text { (overexpression) }\end{array}$ & EML4-ALK (fusion) & 1 & Crizotinib & 8.00 & 7.60 & 7.86 \\
\hline 8 & 1 & $\begin{array}{l}\text { ERBB2 (copy gain, } \\
\text { p.Y772A775dup), } \\
\text { CD274 (copy gain, } \\
\text { overexpression) }\end{array}$ & $\begin{array}{l}\text { ERBB2 (copy gain, } \\
\text { p.Y772A775dup), } \\
\text { CD274 (copy gain, } \\
\text { overexpression) }\end{array}$ & 1 & Afatinib & 8.33 & 5.13 & 1.04 \\
\hline 9 & 3 & ERBB3 (p.P599S) & ERBB3 (p.P599S) & 1 & Afatinib & 26.17 & 8.67 & 4.19 \\
\hline 10 & 0 & EGFR (p.L858R) & EGFR (p.L858R) & 1 & Gefitinib & 80 & 6.43 & N/A \\
\hline 11 & 0 & $\begin{array}{l}\text { ERBB2 (copy gain, } \\
\text { p.Y772A775dup), }\end{array}$ & $\begin{array}{l}\text { ERBB2 (copy gain, } \\
\text { p.Y772A775dup), }\end{array}$ & 1 & Afatinib & 40.27 & 2.10 & N/A \\
\hline 12 & 2 & $\begin{array}{l}\text { TP53 (p.Q192*), KRAS } \\
\text { (p.G12V), EGFR (copy } \\
\text { gain, overexpression), } \\
\text { MET (copy gain, } \\
\text { overexpression), ERBB3 } \\
\text { (overexpression) }\end{array}$ & $\begin{array}{l}\text { KRAS (p.G12V), EGFR } \\
\text { (copy gain, } \\
\text { overexpression), } \\
\text { MET (copy gain, } \\
\text { overexpression) }\end{array}$ & 1 & Erlotinib & 32.03 & 0.93 & $0.22^{\mathrm{a}}$ \\
\hline 13 & 0 & CCDC6-RET (fusion) & CCDC6-RET (fusion) & 2 & Vandetanib & 4.17 & 5.17 & N/A \\
\hline
\end{tabular}

aOngoing treatment.

should be noted that when a patient received "standard therapy" that was deemed to be POG-informed, the treatment was chosen because of the POG data and analysis, rather than because it was the next treatment a patient would receive without that data. This distinction is particularly relevant when there are several standard-of-care choices, particularly in the first-line setting or for other cancers such as breast, when there are many choices that could be considered standard. 


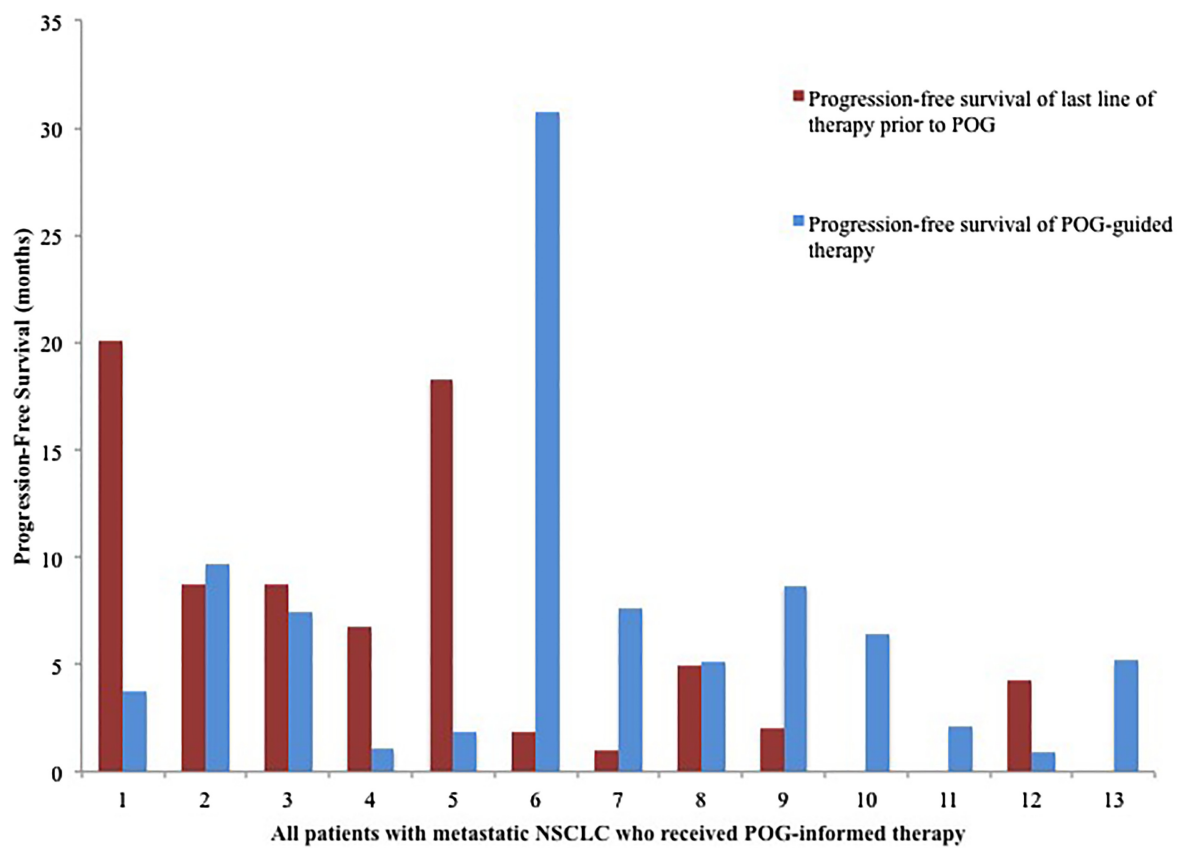

Figure 2. Comparison of progression-free survivals of last line of therapy prior to POG versus POG-guided therapy.

Table 3. Therapies received before POG-informed therapy

\begin{tabular}{|c|c|c|}
\hline Study Patient ID & Histologies & Therapies received before POG \\
\hline 1 & Adenocarcinoma & $\begin{array}{l}\text { Cisplatin/vinorelbine } \\
\text { Cisplatin/etoposide } \\
\text { Erlotinib }\end{array}$ \\
\hline 2 & Adenocarcinoma & Cisplatin/gemcitabine \\
\hline 3 & Adenocarcinoma & Carboplatin/pemetrexed \\
\hline 4 & Squamous cell carcinoma & $\begin{array}{l}\text { Cisplatin/etoposide } \\
\text { Cisplatin/gemcitabine }\end{array}$ \\
\hline 5 & Adenocarcinoma & $\begin{array}{l}\text { Cisplatin/docetaxel } \\
\text { Pemetrexed } \\
\text { Bevacizumab }\end{array}$ \\
\hline 6 & Adenocarcinoma & Cisplatin/pemetrexed \\
\hline 7 & Adenocarcinoma & $\begin{array}{l}\text { Carboplatin/gemcitabine } \\
\text { Pemetrexed/Reolysin } \\
\text { Erlotinib }\end{array}$ \\
\hline 8 & Adenocarcinoma & Cisplatin/gemcitabine \\
\hline 9 & Adenocarcinoma & $\begin{array}{l}\text { Cisplatin/gemcitabine } \\
\text { Pemetrexed } \\
\text { Erlotinib }\end{array}$ \\
\hline 10 & Adenocarcinoma & No prior therapies \\
\hline 11 & Adenocarcinoma & No prior therapies \\
\hline 12 & Adenocarcinoma & $\begin{array}{l}\text { Carboplatin/gemcitabine } \\
\text { Pemetrexed }\end{array}$ \\
\hline 13 & Adenocarcinoma & No prior therapies \\
\hline
\end{tabular}


Table 4. Survival outcomes for patients who received more than one line of POG-informed therapy

\begin{tabular}{|c|c|c|c|c|c|c|c|c|}
\hline \multirow[b]{2}{*}{ Patient } & \multicolumn{4}{|c|}{ First-line POG-informed therapy } & \multicolumn{4}{|c|}{ Second-line POG-informed therapy } \\
\hline & Molecular targets & $\begin{array}{l}\text { POG-informed } \\
\text { therapy }\end{array}$ & PFS & PFS ratio & Molecular targets & POG-informed therapy & PFS & $\begin{array}{l}\mathrm{PFS} \\
\text { ratio }\end{array}$ \\
\hline 3 & $\mathrm{NOTCH} 1$ & Phase $1 \mathrm{NOTCH}$ trial & 7.40 & 0.84 & SDC4-NRG1 fusion & Afatinib & 12.53 & 1.43 \\
\hline 7 & EML4-ALK fusion & Crizotinib & 7.60 & 7.86 & EML4-ALK fusion & $\begin{array}{l}\text { LDK378 (second-generation } \\
\text { ALK inhibitor) }\end{array}$ & 6.73 & 6.97 \\
\hline 8 & ERBB2 (GoF) & Afatinib & 8.67 & 4.19 & CD274 & Nivolumab & 1.20 & 0.24 \\
\hline
\end{tabular}

(GoF) Gain of function.

Ten actionable targets were determined based on DNA analysis, whereas nine targets were based on RNA analysis. In three cases, actionable targets were discovered using RNA analysis, but not detected with DNA. In addition, the EGFR and ALK mutations had previously not been detected as part of routine standard of care, and thus these were included as POG-guided therapies. For instance, for Patient 10, an EGFR mutation was detected on a biopsy from a lesion separate from the previously negative EGFR lung lesion on the initial standard-of-care biopsy done a year prior. For Patient 7, initial standard-of-care testing with immunohistochemistry $(\mathrm{IHC})$ was equivocal (numerical score $2+/ 3$ ) and fluorescent in situ hybridization (FISH) was negative with $3 \%$ positive cells. POG analysis identified an inversion event in Chromosome 2, causing the EML4-ALK fusion gene variant 1, and a translocation with the insertion of a portion of Chromosome 2p containing the EML4-ALK fusion into Chromosome 12 (Fig. 3). The complex nature of two structural variants around the ALK region may be the reason that FISH failed to detect this fusion.
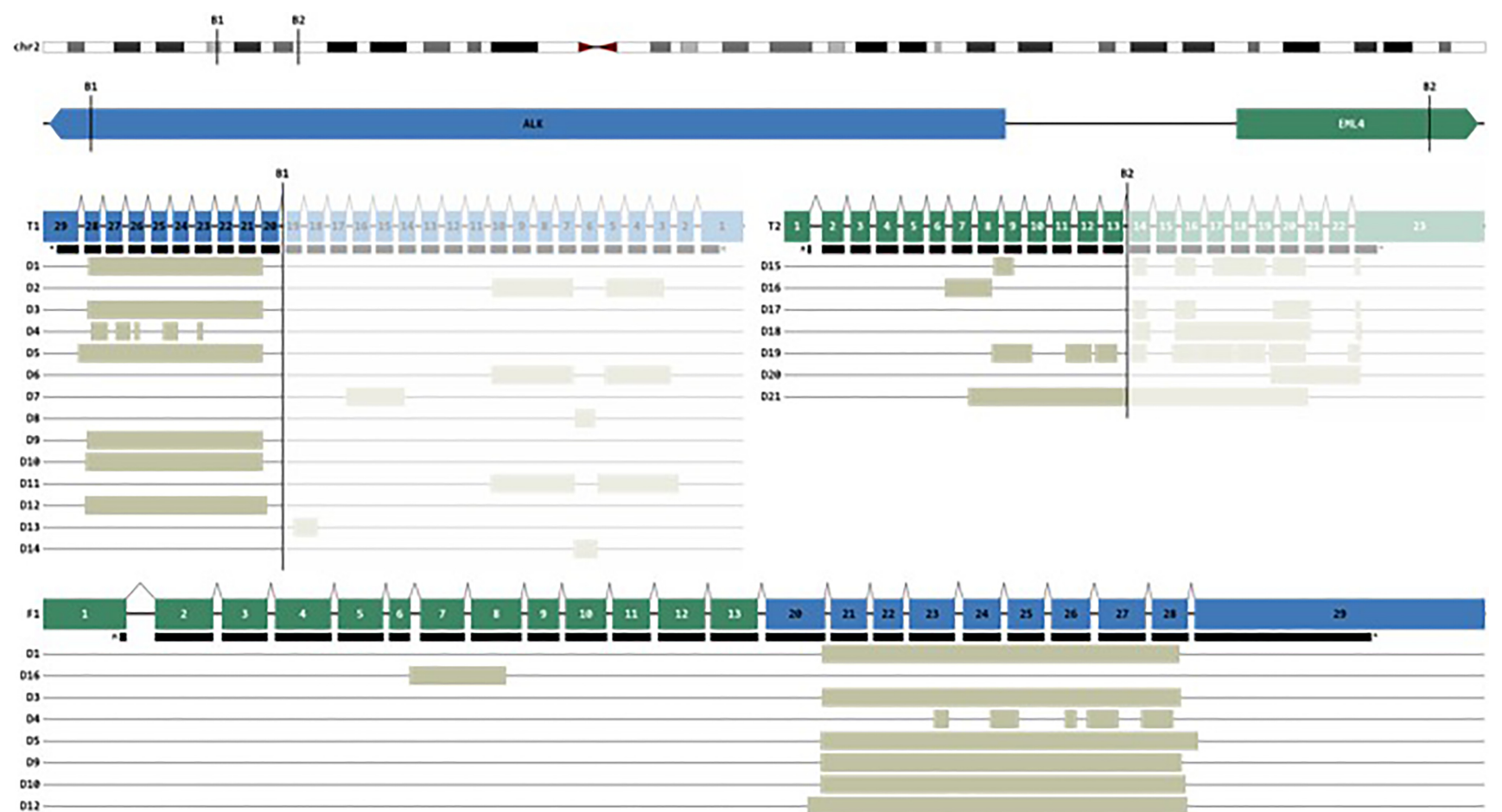

Figure 3. EML4-ALK rearrangement in Patient 7. From the top, fusion breakpoint is shown at the chromosomal, gene, and exon level. Fusion product containing exons 1-13 of EML4 joined to exons 20-29 of ALK is shown on the bottom. 
In our cohort, eight patients received more than one line of therapy that were not guided by POG. Median PFS ratio for this standard therapy cohort was calculated using the most recent therapies and measured 0.84 (IQR: $0.45-1.65$ ). Three (38\%) patients had a PFS ratio $\geq 1.3$, and one (12.5\%) had a PFS ratio $\geq 0.8$ and $<1.3$. Four of these patients had actionable mutations detected from POG analysis, but two declined enrollment in a clinical trial and one patient deteriorated clinically. The fourth patient demonstrated an ALK fusion and high expression of VEGFR on POG analysis. However, this patient was already stable on crizotinib, and thus this was not considered a POG-guided therapy.

\section{DISCUSSION}

Molecular profiling has rapidly come to the forefront of oncology practice, particularly since molecularly targeted agents have demonstrated improved survival outcomes in patients with specific mutations. Our study sought to characterize treatment and outcomes with the addition of a more comprehensive approach to molecular profiling, with whole-genome sequencing and transcriptome analysis.

One of the challenges with genomics-based trials has been defining a clear outcome measure, as patients are not enrolled or treated in a standard randomized or blinded fashion. In our case, the POG program is highly individualized and a simple comparison between PFS of POG-guided therapies would yield little information about the utility of molecular profiling. Thus, the PFS ratio was selected as a surrogate to characterize outcomes, as this marker uses a patient as their own control, comparing PFS with POG-guided therapy to that of a prior systemic therapy regimen. PFS ratio has also been used in other genomics-based trials in oncology (Von Hoff et al. 2010; Tsimberidou et al. 2012; Rodon et al. 2015). In this study, 30\% of patients with metastatic NSCLC and actionable targets had a PFS ratio $\geq 1.3$, indicating a clinical benefit from POG-guided therapy. In a phase II trial also using the PFS ratio as a primary outcome, Von Hoff et al. (2010) used molecular profiling with IHC, FISH, and microarrays to aid in treatment selection for patients with a broad range of metastatic malignancies. A PFS ratio $\geq 1.3$ was defined as a determinant of clinical benefit and, similar to our study, $27 \%$ of patients in their cohort met this cutoff.

In addition, different studies use varying definitions for informative or actionable targets and may have different meanings depending on the type of technology and setting used (Carr et al. 2016). In our study, "actionable" targets were defined as "a potential target or risk factor that affects the treatment plan," and these did not include mutations that are cancer drivers without a clear treatment, such as TP53 mutations. The difference between informative and actionable affects clinical decision-making, and thus a strict definition has been adhered to within our study. As genomic technologies are advancing and increasingly being integrated into patient care, a standardized approach to characterizing actionable mutations will be helpful across cancer centers around the world (Carr et al. 2016).

As the POG trial progressed over time, new evidence became available for pathogenic mutations and the efficacy of novel candidate therapies. Given that our study period spanned from 2012 to 2016, chosen candidate therapies may differ from 2012 compared to 2016, depending on the available evidence at the time. For instance, Patient 9 demonstrated an ERBB3 mutation and was treated with afatinib. At the time of treatment in 2013, De Grève et al. (2012) reported on a number of patients with lung adenocarcinoma who derived clinical benefit with afatinib. Efficacy was later confirmed in a phase II study published in 2015 (De Grève et al. 2015). In another instance, Patient 5 was treated with everolimus and erlotinib based on the available evidence at the time in 2013. There was preclinical evidence of a synergistic benefit of everolimus added to erlotinib to target the rapamycin (mTOR) pathway (Johnson et al. 2007). The phase II study of everolimus and erlotinib 
subsequently published in 2014 did not demonstrate efficacy (Besse et al. 2014). Another example of evolving therapies is the recent availability of immune therapies. Once available, the tumor mutational burden was identified as a marker, and 200 somatic nonsynonymous mutations were used as a cutoff. One of the challenges of these precision medicine trials in clinical oncology is that available therapies may change over the course of the trial, as novel evidence and therapies become available. As a result, the definitions of "actionable" targets may change as well over time, particularly as the treatment landscape evolves. In addition, the data and the patient may have to be taken into account separately, as a particular mutation may be actionable but not necessarily for the patient at the time of genomic analysis.

Despite the changing landscape of precision medicine and its inherent challenges, one of the advantages with whole-genome and RNA data is the ability to retrospectively look back for variants that were previously not known to be actionable. For instance, if a new fusion is discovered, whole-genome and RNA data can easily be queried to find cases with this new fusion. This would not be possible with panel sequencing alone, which can detect only previously known variants. Thus, the ability to reanalyze whole-genome and RNA data is a significant benefit that allows the data to be useful in both the present-day and with future gene discoveries.

The POG program is unique in its comprehensive approach to molecular profiling, with both DNA and RNA sequencing used to determine informative and actionable findings. Previous studies, such as the SHIVA trial, used targeted next-generation sequencing, gene copy-number alterations, and immunohistochemistry for molecular profiling (Le Tourneau et al. 2015). This was a randomized trial that compared outcomes in patients with any solid tumor malignancy that were either matched to a molecularly targeted agent or received treatment chosen by their physician. All of the molecularly targeted agents used were drugs approved for clinical use. No significant difference was determined between the PFS of both groups. In the BATTLE trial, Kim et al. (2011) selected 11 molecular biomarkers to profile patients with metastatic NSCLC and randomized patients to molecularly targeted agents. Molecular testing was limited to 11 biomarkers, and targeted agents were agents being investigated in phase II and III trials for metastatic NSCLC, including erlotinib, vandetanib, erlotinib and bexarotene, and sorafenib. Median PFS was 1.9 mo and disease control rate was $46 \%$ at $8 \mathrm{wk}$. In contrast, the POG program is much broader in its scope of genomic analysis. For instance, the SDC4-NRG1 fusion detected in Patient 3 would not have otherwise been known, and this patient went on to demonstrate a PFS of 12.53 mo with afatinib. The EML4-ALK fusion in Patient 7 also had not been detected with standard FISH. Furthermore, the addition of RNA sequencing may identify genetic aberrations that may not otherwise be found, broadening the potential scope for innovation and discovery of novel treatment strategies. The transcriptome data were useful for a number of practical purposes. It helped to both "validate" the DNA findings (i.e., fusion, mutation) and complement DNA findings. For instance, the genomic data were able to identify an ALK fusion, but transcriptome data can identify the high expression of ALK. In addition, transcriptome data can also detect expression-level aberrations, such as the high expression of targetable genes like EGFR. With whole-genome and RNA data, we are not limited to only known hotspot events but can also better characterize the overall picture of the tumor, providing more context and supporting information to actionable variants. In this relatively small cohort of NSCLC patients, actionable targets were found in three cases with only RNA analysis. Certainly, these remain case examples within a small sample size, and larger studies of the entire POG population will help to determine the incremental benefit of both broad genomic analysis and the addition of RNA sequencing (Laskin et al. 2016).

Prioritizing genomic targets with molecular profiling is another challenge with large-scale genomic analyses, particularly because multiple actionable targets may be identified. Andre 
et al. (2014) proposed a level of evidence scale to aid with target prioritization, ranking molecular alterations validated in early phase trials as highest compared to a predicted target without clinical data as lowest on the scale. In both the SHIVA and BATTLE trials, biomarkers had prespecified rankings to prioritize mutations (Kim et al. 2011; Le Tourneau et al. 2015). In the POG program, despite our relatively small cohort of metastatic NSCLC cases, several patients had multiple actionable molecular targets. Bioinformatics analysis of each case included potential therapies previously reported in the literature that targeted POG-identified pathways. These analyses were presented in a multidisciplinary Clinical Genomics Tumor Board to discuss each actionable target and potential treatments. Previous analysis of the POG program found that the tumor board tended to focus on pathways with several abnormalities, rather than single somatic alterations, to prioritize targets (Laskin et al. 2015). When more than one clinically actionable target is detected and there is clinical equipoise as to the best approach, the decision is left to the patient and their treating oncologist to choose which therapy would be most appropriate. Prospective studies such as the Lung TRACERx, which will use longitudinal sample collection to characterize and investigate intratumor heterogeneity and outcomes in NSCLC, will be helpful in better delineating the changing molecular landscape during disease progression (JamalHanjani et al. 2014). This type of longitudinal data may augment prioritization of molecular targets.

Limitations of our study include our relatively small sample size of metastatic NSCLC cases enrolled in the POG program. Genomic analysis and comparison of PFS ratios in a larger cohort may provide an improved understanding of the broader implications of these results. This largely served as a descriptive study to disseminate our institutional experience with metastatic NSCLC in a genomics-based setting.

\section{CONCLUSION}

In conclusion, we present our institutional experience with integrating whole-genome and RNA sequencing into practice for patients with metastatic NSCLC. Within this relatively small cohort of NSCLC, $30 \%$ of patients who were able to receive treatment demonstrated longer PFS with POG-informed therapies. Our population-based approach highlights the utility and advantages of whole-genome and RNA data over gene panels in informing our knowledge about cancer biology and gene discovery. Larger studies over a longitudinal basis will help clarify the role of whole-genome analysis in routine clinical practice.

\section{METHODS}

\section{Personalized Onco-Genomics Project}

$\mathrm{BC}$ Cancer $(\mathrm{BCC})$ is responsible for coordinating cancer care for the $\sim 4.6$ million residents across British Columbia. The POG program was launched as a translational medicine study to create a multidisciplinary network of oncologists, pathologists, and bioinformaticians to apply whole-genome and transcriptome analysis to guide treatment decision-making for patients with metastatic malignancies when limited standard treatment options were available. Between 2012 and 2016, 217 patients had a fresh tumor biopsy and blood sample (to differentiate germline aberrations). The tumor samples were classified according to the World Health Organization criteria. Samples underwent comprehensive DNA $(80 \times$ for tumor samples; $40 \times$ for normal DNA comparator) and RNA sequencing (200 million reads) followed by in-depth bioinformatics, including the assembly, annotation, and mining of genomic data to identify abnormalities that might be cancer "drivers" or provide actionable or treatable 
targets. Each case was presented and discussed in a weekly multidisciplinary molecular tumor board during which the consenting/treating medical oncologist and colleagues interacted directly with the team of genome scientists and analysts to gain a comprehensive and mutually educational understanding of the genomic findings for this individual with incurable cancer. During this meeting, "informative" targets were defined as an interesting feature that may or may not have prognostic or therapeutic relevance at this time but play a role in better understanding the biology or diagnosis of the cancer. Most of these targets were felt to play a role in carcinogenesis but had no available agents to affect the target or pathway at this time. For instance, cancer drivers, such as TP53 mutations, were included as informative targets. In contrast, "clinically actionable" targets were considered a potential target or risk factor that affects the treatment plan (Laskin et al. 2015; Massard et al. 2017). The average time to results from biopsy measured $54.5 \mathrm{~d}$. The length of time is primarily because of the in-depth personalized genome and transciptome analysis that is undertaken by a highly specialized team of bioinformaticians. This adds to the time; however, it also means that the data and the body of evidence to support any genomic target has been thoroughly explored, doing justice to the sheer volume and complexity of data that is generated for each individual patient. As the program has evolved over time, a manually curated Knowledgebase of genomic data has been assembled. This turnaround time is improving, but it is not expected to diminish rapidly or negate a personalized evaluation and analysis. POG has integrated a more time-sensitive, automated "targeted gene report" that is a first look at the whole-genome and transcriptome data with a preselected set of well-defined targets. The average turnaround time for this "targeted report" is $26 \mathrm{~d}$. This manuscript is focused on comprehensive analyses rather than this targeted report. Further details regarding sequencing and bioinformatics are described in Supplemental Appendix 1.

The POG project was approved by the University of British Columbia Cancer Research Ethics Board (BCCA REB ID: H14-00681) and conducted in accordance with the Declaration of Helsinki. Informed consent was obtained from the patient for profiling the tumor using RNA and whole-genome sequencing.

\section{Patient Population}

For this study, we identified all patients enrolled in the POG program between 2012 and 2016 who had been diagnosed with metastatic NSCLC. Histologies included were adenocarcinoma, squamous cell carcinoma, and NSCLC-not otherwise specified (NSCLC-NOS). Baseline clinicopathologic and treatment data were extracted by retrospective chart review, including Eastern Cooperative Oncology Group (ECOG) performance status, treatment modalities, and outcomes. ECOG status is a standard measure of a patient's functional status that is commonly used in clinical trials (Oken et al. 1982). "Informative" and "actionable" targets were identified based on POG analysis, with detailed descriptions of potential pathways, along with the level of evidence behind candidate therapies and potential targets. The majority of cases included in the POG program were presented and discussed in the weekly Clinical Genomics Tumor Board, which included medical oncologists, pathologists, and bioinformaticians. A consensus of the Clinical Genomics Tumor Board, including the patient's treating clinician, was reached to identify each informative and clinically actionable target. Decisions regarding "actionable" targets and appropriate therapies were prioritized according to levels of evidence (Table 5), with known alterations and approved therapies ranking higher than those with emerging evidence. "POG-informed therapy" was defined as a treatment chosen based on an actionable target determined by POG analysis. Therapies based on standard-of-care mutation analysis, such as EGFR mutations, were not considered "POG-informed therapies," unless this was not previously known, as the POG data are intended for discovery and innovation and not to replace a routine gene test. 
Table 5. Tiers of evidence utilized by the Clinical Genomics Tumor Board when prioritizing actionable mutations and potential therapies

\begin{tabular}{ll}
\hline Tier & \multicolumn{1}{c}{ Actionable alteration and levels of evidence } \\
\hline 1 & Known alterations with approved drug in same cancer type \\
2 & Known alterations with approved therapy in different cancer types \\
3 & Known alterations with emerging therapeutic evidence \\
4 & $\begin{array}{c}\text { Uncharacterized oncologic alterations identified by POG without existing evidence based } \\
\text { on current literature }\end{array}$ \\
\hline
\end{tabular}

Overall survival (OS) data were calculated using the date of diagnosis, whereas PFS on therapy was calculated using the start date of therapy.

\section{Statistical Analysis}

Descriptive statistics were calculated to characterize our series of patients with metastatic NSCLC who underwent whole-genome and RNA sequencing through the POG program. Categorical variables were compared using Pearson's $\chi^{2}$ test. PFS ratio was utilized as a surrogate to characterize outcomes where the patient acts as their own control, similar to other genomics-based trials (Von Hoff et al. 2010). We calculated the PFS ratio by comparing the PFS of POG-informed therapies with the PFS of the last regimen prior to POG. OS was calculated from the date of diagnosis to date of death or last follow-up. All tests were two-sided, with $P \leq 0.05$ as the cutoff for statistical significance. SPSS version 22.0 was used for all statistical analyses (SPSS).

\section{ADDITIONAL INFORMATION}

\section{Data Deposition and Access}

The whole-genome sequencing and RNA-seq data for this case are available as .bam files from the European Genome-Phenome Archive (EGA, www.ebi.ac.uk/ega/home) as part of the study EGAS00001001159, accession IDs include EGAD00001001963, EGAD00001002045, EGAD00001001965，EGAD00001001967，EGAD00001001968，EGAD00001002985, EGAD00001001969，EGAD00001001966，EGAD00001001962，EGAD00001002564, EGAD00001003011，EGAD00001002023，EGAD00001002548，EGAD00001001964, EGAD00001003005, EGAD00001001961, EGAD00001003047, EGAD00001003057, and EGAD00001003076.

\section{Ethics Statement}

The study was approved by the University of British Columbia Research Ethics Committee (BCCA REB ID: H12-00137) and conducted in accordance with the Declaration of Helsinki. Written informed consent was obtained from each patient prior to genomic profiling. Patient identification was anonymized within the research team and an identification code was assigned to the case for communicating clinically relevant information to physicians. The patients consented to potential publication of findings. Raw sequence data and downstream analytics were maintained within a secure computing environment.

\section{Acknowledgments}

We gratefully acknowledge the participation of our patients and families, the POG team, and the generous support of the BC Cancer Foundation. We also acknowledge contributions 
Competing Interest Statement

The authors have declared no competing interest.

Received January 14, 2018; accepted in revised form November 5, 2018. toward equipment and infrastructure from Canada Foundation for Innovation and the BC Knowledge Development Fund. The results published here are in part based upon data generated by the following projects and obtained from dbGaP (http://www.ncbi.nlm.nih.gov/ gap): The Cancer Genome Atlas managed by the National Cancer Institute ( $\mathrm{NCl}$ ) and the National Human Genome Research Institute (NHGRI) (http://cancergenome.nih.gov).

\section{Author Contributions}

J.L., E.S.T., Y.S., N.C., and C.H. were responsible for the concept, design, and analysis for this study. All authors discussed the results and contributed to the final manuscript.

\section{Funding}

This research did not receive any specific grant from funding agencies in the public, commercial, or not-for-profit sectors.

\section{REFERENCES}

Andre F, Nowak F, Arnedos M, Lacroix L, Viens P, Calvo F. 2012. Biomarker discovery, development, and implementation in France: a report from the French National Cancer Institute and cooperative groups. Clin Cancer Res 18: 1555-1560. doi:10.1158/1078-0432.CCR-11-2201

Andre F, Mardis E, Salm M, Soria JC, Siu LL, Swanton C. 2014. Prioritizing targets for precision cancer medicine. Ann Oncol 25: 2295-2303. doi:10.1093/annonc/mdu478

Besse B, Leighl N, Bennouna J, Papadimitrakopoulou VA, Blais N, Traynor AM, Soria JC, Gogov S, Miller N, Jehl V, et al. 2014. Phase II study of everolimus-erlotinib in previously treated patients with advanced non-small-cell lung cancer. Ann Oncol 25: 409-415. doi:10.1093/annonc/mdt536

Canadian Cancer Society. 2016. Canadian Cancer Statistics 2016. [Internet]; c2016 [cited 2017 March 20]. http://www.cancer.ca/ /media/cancer.ca/CW/cancer\%20information/cancer\%20101/Canadian\%20cancer\% 20statistics/Canadian-Cancer-Statistics-2016-EN.pdf?la=en

Carr TH, McEwen R, Dougherty B, Johnson JH, Dry JR, Lai Z, Ghazoui Z, Laing NM, Hodgson DR, Cruzalegui F, et al. 2016. Defining actionable mutations for oncology therapeutic development. Nat Rev Cancer 16: 319-329. doi:10.1038/nrc.2016.35

De Grève J, Teugels E, Geers C, Decoster L, Galdermans D, De Mey J, Everaert H, Umelo I, In't Veld P, Schallier D. 2012. Clinical activity of afatinib (BIBW 2992) in patients with lung adenocarcinoma with mutations in the kinase domain of HER2/neu. Lung Cancer 76: 123-127. doi:10.1016/j.lungcan.2012.01.008

De Grève J, Moran T, Graas MP, Galdermans D, Vuylsteke P, Canon JL, Schallier D, Decoster L, Teugels E، Massey D, et al. 2015. Phase II study of afatinib, an irreversible ErbB family blocker, in demographically and genotypically defined lung adenocarcinoma. Lung Cancer 88: 63-69. doi:10.1016/j.lungcan.2015 .01 .013

Jamal-Hanjani M, Hackshaw A, Ngai Y, Shaw J, Dive C, Quezada S, Middleton G, de Bruin E, Le Quesne J, Shafi S, et al. 2014. Tracking genomic cancer evolution for precision medicine: the Lung TRACERx study. PLOS Biol 12: e1001906. doi:10.1371/journal.pbio.1001906

Johnson BE, Jackman D, Jänne PA. 2007. Rationale for a phase I trial of erlotinib and the mammalian target of rapamycin inhibitor everolimus (RAD001) for patients with relapsed non-small cell lung cancer. Clin Cancer Res 13: s4628-s4631. doi:10.1158/1078-0432.CCR-07-0717

Johnson DH, Schiller JH, Bunn PA Jr. 2014. Recent clinical advances in lung cancer management. J Clin Oncol 32: 973-982. doi:10.1200/JCO.2013.53.1228

Kim ES, Herbst RS, Wistuba II, Lee JJ, Blumenschein GR Jr, Tsao A, Stewart DJ, Hicks ME, Erasmus J Jr, Gupta $\mathrm{S}$, et al. 2011. The BATTLE trial: personalizing therapy for lung cancer. Cancer Discov 1: 44-53. doi: 10.1158/2159-8274.CD-10-0010

Laskin J, Jones S, Aparicio S, Chia S, Ch'ng C, Deyell R, Eirew P, Fok A, Gelmon K, Ho C, et al. 2015. Lessons learned from the application of whole-genome analysis to the treatment of patients with advanced cancers. Cold Spring Harb Mol Case Stud 1: a000570. doi:10.1101/mcs.a000570

Laskin J, Ho C, Shen Y, Jones M, Gelmon KA, Lim H, Renouf DJ, Yip S, Tinker A, Khoo K, et al. 2016. Availability of tumour gene expression data facilitates clinical decision-making for patients with advanced cancers. Ann Oncol 27: 526-544. doi:10.1093/annonc/mdv591

Le Tourneau C, Delord JP, Gonçalves A, Gavoille C, Dubot C, Isambert N, Campone M, Trédan O, Massiani MA, Mauborgne C, et al. 2015. Molecularly targeted therapy based on tumour molecular profiling versus 
conventional therapy for advanced cancer (SHIVA): a multicentre, open-label, proof-of-concept, randomised, controlled phase 2 trial. Lancet Oncol 16: 1324-1334. doi:10.1016/S1470-2045(15)00188-6

Lindsay CR, Shaw E, Walker I, Johnson PW. 2015. Lessons for molecular diagnostics in oncology from the cancer research UK stratified medicine programme. Expert Rev Mol Diagn 15: 287-289. doi:10.1586/ 14737159.2015.992417

MacConaill LE, Garraway LA. 2010. Clinical implications of the cancer genome. J Clin Oncol 28: 5219-5228. doi:10.1200/JCO.2009.27.4944

Massard C, Michiels S, Ferté C, Le Deley MC, Lacroix L, Hollebecque A, Verlingue L, lleana E, Rosellini S, Ammari S, et al. 2017. High-throughput genomics and clinical outcome in hard-to-treat advanced cancers: results of the MOSCATO 01 trial. Cancer Discov 7: 586-595. doi:10.1158/2159-8290.CD-16-1396

Oken MM, Creech RH, Tormey DC, Horton J, Davis TE, McFadden ET, Carbone PP. 1982. Toxicity and response criteria of the Eastern Cooperative Oncology Group. Am J Clin Oncol 5: 649-655. doi:10.1097/ 00000421-198212000-00014

Rodon J, Soria JC, Berger R, Batist G, Tsimberidou A, Bresson C, Lee JJ, Rubin E, Onn A, Schilsky RL, et al. 2015. Challenges in initiating and conducting personalized cancer therapy trials: perspectives from WINTHER, a Worldwide Innovative Network (WIN) Consortium trial. Ann Oncol 26: 1791-1798. doi: 10.1093/annonc/mdv191

Siegel RL, Miller KD, Jemal A. 2016. Cancer statistics 2016. CA Cancer J Clin 66: 7-30.

Simon R, Roychowdhury S. 2013. Implementing personalized cancer genomics in clinical trials. Nat Rev Drug Discov 12: 358-369. doi:10.1038/nrd3979

Stratton MR, Campbell PJ, Futreal PA. 2009. The cancer genome. Nature 458: 719-724. doi:10.1038/ nature 07943

Tsimberidou AM, Iskander NG, Hong DS, Wheler JJ, Falchook GS, Fu S, Piha-Paul S, Naing A, Janku F, Luthra $\mathrm{R}$, et al. 2012. Personalized medicine in a phase I clinical trials program: the MD Anderson Cancer Center Initiative. Clin Cancer Res 18: 6373-6383. doi:10.1158/1078-0432.CCR-12-1627

Von Hoff DD, Stephenson JJ Jr, Rosen P, Loesch DM, Borad MJ, Anthony S, Jameson G, Brown S, Cantafio N, Richards DA, et al. 2010. Pilot study using molecular profiling of patients' tumors to find potential targets and select treatments for their refractory cancers. J Clin Oncol 28: 4877-4883. doi:10.1200/JCO 2009.26 .5983 